\title{
PHOTO- AND ELECTROPRODUCTION OF KAONS
}

\author{
P. BYDŽOVSKÝ \\ Nuclear Physics Institute, 25068 Řez near Prague, Czech Republic
}

\begin{abstract}
Isobar models for the electromagnetic production of $\mathrm{K}^{+}$and $\mathrm{K}^{0}$ are discussed with emphasis on the $\mathrm{K}^{0}$ photoproduction off neutron. Predictions of the models for the $\mathrm{K}^{0}$ photoproduction on deuteron are compared with the recent data and some properties of the elementary amplitudes are shown.
\end{abstract}

\section{Introduction}

The electromagnetic production of strangeness on nucleon provides us with additional information about the structure and interactions of baryons. Beyond investigation of the reaction mechanism, form factors of hadrons and new "missing" resonances one also needs a correct description of the process for a study of more complex processes such as the electroproduction of hypernuclei. A good quality description of the elementary process minimises uncertainty in calculations of the excited spectra for the hypernucleus electroproduction [1]. Many new good quality experimental data for the $\mathrm{K}^{+}$production collected in JLab, ELSA, SPring-8, and GRAAL allow performing a thorough analysis of the elementary process. Moreover, the recent data on the photoproduction of $\mathrm{K}^{0}$ off deuteron from Tohoku University [2] facilitate doing more rigorous tests of elementary amplitudes $[2,3]$.

The electroproduction process can be formally reduced to investigation of the binary process of the photoproduction by virtual photons since the electromagnetic coupling constant is small enough to justify the one-photon approximation. There are several approaches to treat the photoproduction process. Among them the isobar models based on the effective Lagrangian description considering only the hadronic degrees of freedom are suitable for their further use in the more complex calculations. Other approaches are eligible either for higher energies $\left(E_{\gamma}>4 \mathrm{GeV}\right)$, the Regge model [4], or to the threshold region, the Chiral Perturbation Theory [5]. Quark models [6] are too complicated for their further use in the hypernuclear calculations. Another approach, aimed at the forward-angle production, is the hybrid Regge-plus-resonance model [7] in which the background part of amplitude 
is generated by the $t$-channel Regge-trajectory exchange and the resonant behaviour is shaped by the s-channel resonances like in an isobar model.

\section{Isobar Models}

In the effective hadron Lagrangian approach, various channels connected via the final-state interaction (the meson-baryon rescattering processes) have to be treated simultaneously to take unitarity properly into account $[8,9]$. In Ref. 9 the coupled-channel approach has been used to include effects of the $\pi \mathrm{N}$ intermediate states in the $\mathrm{p}\left(\gamma, \mathrm{K}^{+}\right) \Lambda$ process. However, tremendous simplifications originate in neglecting the rescattering effects in the formalism assuming that they are included to some extent by means of effective values of the strong coupling constants fitted to data. This simplifying assumption was adopted in many of the isobar models, e.g., Saclay-Lyon (SLA) [10], Kaon-MAID (KM) [11], and Janssen et al. [12].

In isobar models, the amplitude obtains contributions from the Born terms and exchanges of resonances. Due to absence of a dominant resonance in the process (unlike in the $\pi$ and $\eta$ photoproduction) large number of possible combinations of the resonances with mass smaller than $2 \mathrm{GeV}$ must be taken into account [10]. This number of models is limited considering constraints set by $\mathrm{SU}(3)[10,11]$ and crossing symmetries $[10,13]$ and by duality hypothesis [13]. Adopting the SU(3) constraints to the two main coupling constants, however, makes the contribution of the Born terms nonphysically large [12]. To reduce this contribution, either hyperon resonances [10] or hadron form factors [11] must be added, or a combination of both [12]. The hadron form factors which simulate a structure in the strong vertex are included in the KM and Janssen models maintaining the gauge invariance of amplitude $[11,12]$. Here we use the KM and SLA models.

The strong coupling constants in the $\mathrm{K}^{0} \Lambda$ and $\mathrm{K}^{+} \Lambda$ channels are related via the $\mathrm{SU}(2)$ isospin symmetry: $g_{\mathrm{K}+\Lambda \mathrm{p}}=g_{\mathrm{K}^{0} \Lambda \mathrm{n}}$ and $g_{\mathrm{K}+\Sigma^{0} \mathrm{p}}=-g_{\mathrm{K}^{0} \Sigma^{0} \mathrm{n}}$. In the electromagnetic vertexes a ratio of the neutral to charged coupling constants have to be known. For the nucleon and its resonances the ratio can be related to the known helicity amplitudes of the nucleons [14] whereas for the kaon resonances the ratio relates to the decay widths known only for the $\mathrm{K}^{*}$ meson [14]: $\mathrm{r}_{\mathrm{K}^{*}}=-\sqrt{\Gamma_{\mathrm{K}^{* 0} \rightarrow \mathrm{K}^{0} \gamma} / \Gamma_{\mathrm{K}^{*+} \rightarrow \mathrm{K}^{+} \gamma}}=-1.53$ where the sign was set from the quark model prediction. Since the decay widths of the $\mathrm{K}_{1}$ meson are unknown the appropriate ratio, $\mathrm{r}_{\mathrm{K}_{1}}$, have to be fixed in the models. It was fitted to the $\mathrm{K}^{0} \Sigma^{+}$data in $\mathrm{KM}[11], \mathrm{r}_{\mathrm{K}_{1}}=-0.45$, but it is a free parameter in SLA (see also Refs. 2 and 3). For hyperons, the electromagnetic vertex is not changed in the $\mathrm{K}^{0} \Lambda$ channel. 


\section{Photoproduction on the Deuteron}

The inclusive cross section for the $\mathrm{d}\left(\gamma, \mathrm{K}^{0}\right) \mathrm{YN}^{\prime}$ or $\mathrm{d}(\gamma, \Lambda) \mathrm{KN}^{\prime}$ process ( $\mathrm{Y}$ stands for $\Lambda$ or $\Sigma$ and N' for proton or neutron) in the threshold region is calculated in the impulse approximation in which the nucleon N' acts as a spectator [3]. Contributions of the final-state interaction (FSI) to the inclusive cross section were shown to be small in the studied kinematical region [15]. Moreover, we assume that a part of the KY rescattering effects is absorbed in the coupling constants of the elementary amplitude and that the $\mathrm{KN}$ interaction is weak on the hadronic scale. The main lack of precision, therefore, comes from ignoring the YN FSI.

In the calculations we have chosen the off-shell approximation [3] in which the four-momentum is conserved in the production vertex forcing the target nucleon off its mass shell. The momentum distribution of the target nucleon is described by the nonrelativistic Bonn deuteron wave function OBEPQ [16]. More details about the calculations can be found in Ref. 3.

\section{Results and Discussion}

The existing isobar models do not give satisfactory description of the new data on the $\mathrm{K}^{+}$photoproduction, especially of the spin observables [17]. Here we mention only the difference in results of the models for the forward kaon-angle cross section which causes substantial uncertainty in predictions for the hypernucleus photoproduction cross sections [1]. At very forward angle, $\theta_{\mathrm{K}}^{\text {lab }}=3^{\circ}$, and photon lab energy $1.42 \mathrm{GeV}$, the lab cross sections predicted by the SLA and KM models are 2.24 and $1.60 \mu \mathrm{b} / \mathrm{sr}$, respectively. This $30 \%$ discrepancy gives corresponding difference in results for the photoproduction of ${ }^{12} \mathrm{~B}_{\Lambda}$ at $1.42 \mathrm{GeV}$ : the excitation cross sections for the two main multiplets $(\Lambda$ in $s$ and $p$ state is coupled to the ground state of the core nucleus ${ }^{11} \mathrm{~B}$ ) are 169 and $173 \mathrm{nb} / \mathrm{sr}$ for SLA and 119 and $127 \mathrm{nb} / \mathrm{sr}$ for KM, respectively. We see that the elementary amplitude needs to be better fixed at forward angles to give reliable results for the hypernucleus calculations. Studying the process in other channels, e.g., $\mathrm{n}\left(\gamma, \mathrm{K}^{0}\right) \Lambda$, might help in this respect.

In Figure 1 results for the inclusive momentum spectra of the $\mathrm{d}\left(\gamma, \mathrm{K}^{0}\right) \Lambda \mathrm{p}$ process calculated with different elementary amplitudes are shown in comparison with the recent data from LNS of Tohoku University [2]. Contributions of the $\Sigma$ channels are very small in the lower-energy region (l.e.r.) (Fig. 1a) and at the spectator-kinematics region (the main peak) in Fig. 1b [2]. The KM model do not describe the data well, especially at 

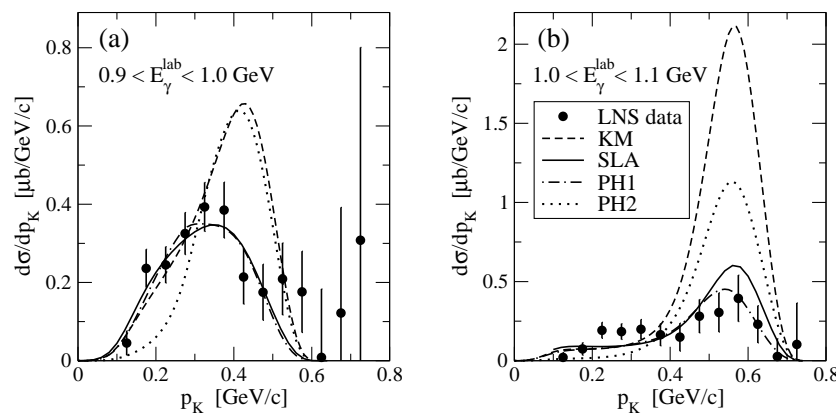

Fig. 1. Inclusive energy-averaged, (a) $0.9<\mathrm{E}<1.0 \mathrm{GeV}$ and (b) $1.0<\mathrm{E}<1.1 \mathrm{GeV}$, and kaon-angle-integrated, $0.9<\cos \left(\theta_{K}\right)<1.0$, momentum spectra for the $\mathrm{d}\left(\gamma, \mathrm{K}^{0}\right) \mathrm{YN}^{\prime}$ process. Calculations using various elementary amplitudes are performed for the $\Lambda \mathrm{p}$ final state. Data are from Ref. 2.

the spectator-kinematics region. Results of KM cannot be improved even if the $\mathrm{r}_{\mathrm{K}_{1}}$ parameter is fitted to the l.e.r. data: $\chi_{\text {n.d.f. }}^{2}=3.64$ with $\mathrm{r}_{\mathrm{K}_{1}}=-2.34$. The SLA model with $\mathrm{r}_{\mathrm{K}_{1}}=-2.09$, fitted to the l.e.r. data $\left(\chi_{\text {n.d.f. }}^{2}=0.88\right)[2]$, gives good results in both energy regions. The angular dependence of the elementary cross sections differs for these models as shown in Fig. 2. To point out importance of the angular dependence of cross section the phenomenological prescriptions PH1 and PH2 (see Ref. 2 for details) were used. PH1 which possesses the backward-peaked cross section, Fig. 2, gives much better results for $\mathrm{d}\left(\gamma, \mathrm{K}^{0}\right) \Lambda \mathrm{p}$ than $\mathrm{PH} 2$ with the opposite angular dependence (Fig. 2). This comparison shows that the Tohoku data prefer models which give a backward-peaked cross section for the $\mathrm{n}\left(\gamma, \mathrm{K}^{0}\right) \Lambda$ reaction.

Significance of various contributions in the elementary cross section at $E_{\gamma}=1.1 \mathrm{GeV}$ is shown for SLA and KM in Fig. 3. If the contribution of
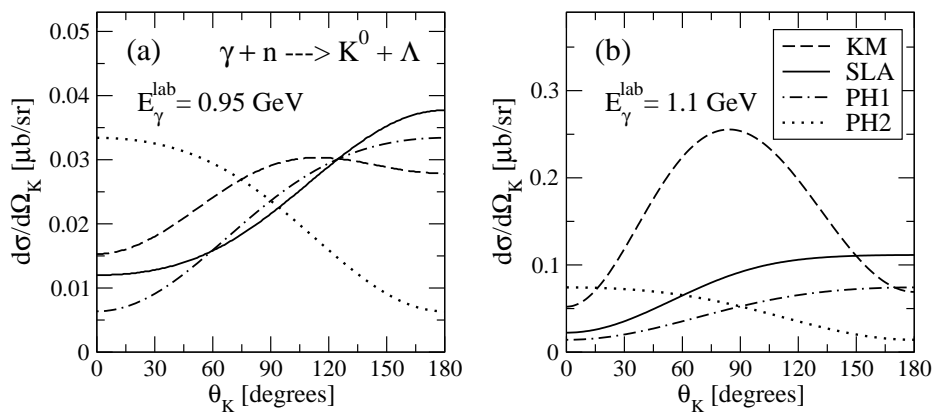

Fig. 2. Predictions of the models for the c.m. differential cross section in the photoproduction of $\mathrm{K}^{0}$ on neutron are shown for the photon lab energies 0.95 and $1.1 \mathrm{GeV}$. 

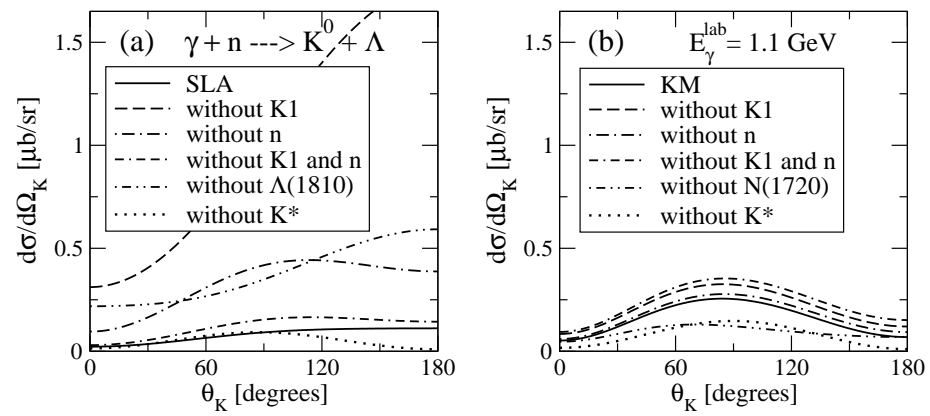

Fig. 3. Angular dependence of the c.m. cross section for the photoproduction of $\mathrm{K}^{0}$ on neutron at $1.1 \mathrm{GeV}$. Results for the Saclay-Lyon (a) and Kaon-MAID (b) models are shown when some of the contributions are switched off.

neutron or $\mathrm{K}_{1}$ is omitted in SLA the cross sections change largely, Fig. 3a, whereas the omission of both terms simultaneously makes a very small effect. This shows that the main role of $K_{1}$, with the fitted parameter $r_{K_{1}}$, is to compensate the neutron contribution. This phenomenon is absent in KM, Fig. 3b, since the neutron contribution itself is already strongly suppressed by the hadron form factor. In both models the contribution of $\mathrm{K}^{*}$ is important at large $\theta_{\mathrm{K}}$. The hyperon resonances, included in SLA but not in KM, also contribute notably at backward angles (Fig. 3a). The nucleon resonances give a negligible contribution at $\theta_{\mathrm{K}} \approx 180^{\circ}$ in both models.

In Figure 4 predictions of the KM and SLA models for the momentum spectra of $\mathrm{d}(\gamma, \Lambda) \mathrm{K}^{+} \mathrm{n}$ and $\mathrm{d}(\gamma, \Lambda) \mathrm{K}^{0} \mathrm{p}$ (a) are shown with the angular dependence of the elementary cross sections (b). The models give signifi-
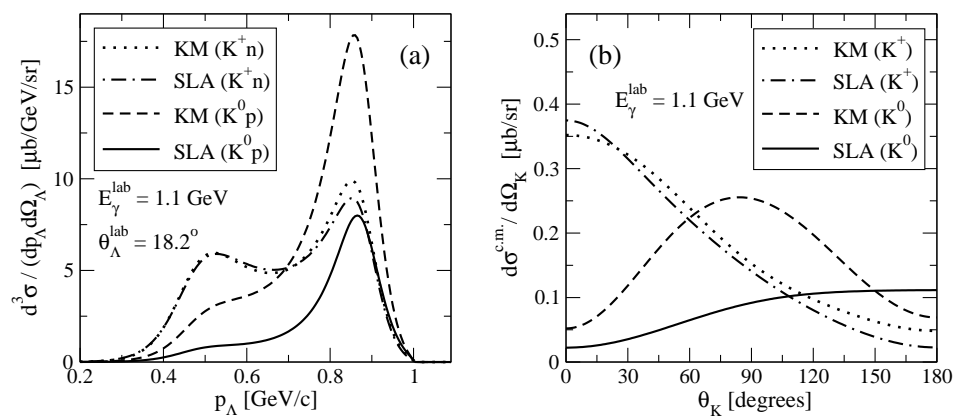

Fig. 4. Predictions of the Saclay-Lyon (SLA) and Kaon-MAID (KM) models for (a) momentum spectra of the $\Lambda$ photoproduction on deuteron with $\mathrm{K}^{+} \mathrm{n}$ and $\mathrm{K}^{0} \mathrm{p}$ final states; (b) angular dependence of the c.m. cross section for the $\Lambda$ photoproduction on the proton and neutron with associated $\mathrm{K}^{+}$and $\mathrm{K}^{0}$ in the final state, respectively. 
cantly different results for the $\mathrm{K}^{0}$ production whereas they accord for the $\mathrm{K}^{+}$channel. This shows that the $\mathrm{K}^{0}$ photoproduction on deuteron provides with additional information about dynamics of the elementary process.

To summarise: the isobar models for the $\mathrm{K}^{+}$photoproduction need revision at forward kaon angles before they can give reliable predictions for the hypernuclear calculations. The data on the $\mathrm{d}\left(\gamma, \mathrm{K}^{0}\right) \mathrm{YN}$ ' reaction near the threshold clearly prefer the models for the $\mathrm{n}\left(\gamma, \mathrm{K}^{0}\right) \Lambda$ reaction which give enhancement of the cross section at the backward kaon angles. These data can, therefore, discriminate between the models which otherwise fit the $\mathrm{p}\left(\gamma, \mathrm{K}^{+}\right) \Lambda$ data equally well.

The author wishes to thank the organisers for their kind invitation to this highly stimulating Symposium. This work was supported by the Grant Agency of the Czech Republic, grant 202/08/0984.

\section{References}

1. P. Bydžovský, M. Sotona, T. Motoba, K. Itonaga, K. Ogawa, and O. Hashimoto, arXiv:0706.3836 [nucl-th].

2. K. Tsukada et al., Phys. Rev. C 78, 014001 (2008).

3. P. Bydžovský, M. Sotona, O. Hashimoto, and T. Takahashi, nucl-th/0412035.

4. M. Guidal, J.-M. Laget, M. Vanderhaeghen, Nucl. Phys. A 627, 645 (1997).

5. S. Steininger and U.-G. Meissner, Phys. Lett. B 391, 446 (1997).

6. B. Saghai, in Proc. of Electrophotoproduction of Strangeness on Nucleons and Nuclei, Sendai, Japan, 16-18 June, 2003, (Eds. K.Maeda, H.Tamura, S.N.Nakamura, O.Hashimoto). World Sci., 2004, p.53; nucl-th/0310025.

7. T. Corthals, J. Ryckebusch, and T. Van Cauteren, Phys. Rev. C 73, 045207 (2006); T. Corthals, T. Van Cauteren, P. Van Craeyveld, J. Ryckebusch, and D.G. Ireland, Phys. Lett. B 656, 186 (2007).

8. G. Penner and U. Mosel, Phys. Rev. C 66, 055212 (2002); A. Usov and O. Scholten, Phys. Rev. C 72, 025205 (2005).

9. W.T. Chiang, F. Tabakin, T.-S.H. Lee, B. Saghai, Phys. Lett. B 517, 101 (2001).

10. J.C. David, C. Fayard, G.-H. Lamot, B. Saghai, Phys. Rev. C 53, 2613 (1996); T. Mizutani, C. Fayard, G.-H. Lamot, B. Saghai, Phys. Rev. C 58, 75 (1998).

11. T. Mart and C. Bennhold, Phys. Rev. C 61, 012201 (2000); C. Bennhold et al., nucl-th/9901066.

12. S. Janssen, J. Ryckebusch, D. Debruyne, and T. Van Cauteren, Phys. Rev. C 65, 015201 (2001).

13. R.A.Williams, Chueng-Ryong Ji, S.R.Cotanch, Phys. Rev. C 46, 1617 (1992).

14. C. Amsler et al. (Particle Data Group), Phys. Lett. B 667, 1 (2008).

15. A. Salam, K. Miyagawa, T. Mart, C. Bennhold and W. Glöckle, Phys. Rev. C 74, 044004 (2006).

16. R. Machleidt, K. Holinde, and Ch. Elster, Phys. Rep. 149, 1 (1987).

17. R.K. Bradford et al., Phys. Rev. C 75, 035205 (2007). 